\title{
RAZÃO CINTURA-ESTATURA COMO DISCRIMINADOR DO RISCO CORONARIANO DE ADULTOS
}

\section{Francisco José Gondim Pitanga*, Ines Lessa}

Trabalho realizado no ISC (Instituto de Saúde Coletiva) e na UFBA (Universidade Federal da Bahia)

\section{*Correspondência:}

Av. Luiz Tarquínio Pontes, 600, Centro

Cep: 42.700-00, Lauro de Freitas/BA

pitanga@lognet.com.br

\section{RESUMO}

Oвjetivo. Selecionar, por meio da sensibilidade e especificidade, os melhores pontos de coorte para a razão cintura-estatura (RCEst) como discriminador de risco coronariano elevado (RCE).

Métodos. $O$ desenho foi transversal com amostra composta por 968 adultos de 30-74 anos de idade, sendo 39I (40,4\%) do sexo masculino. A análise foi feita por curva Receiver Operating Characteristic (ROC) para identificar a sensibilidade e especificidade do melhor ponto de coorte da RCEst como discriminador de RCE. Verificou-se também a significância estatística da área sob a curva ROC. Foi utilizado intervalo de confiança (IC) a 95\%.

Resultados. A área total sob a curva ROC entre RCEst e RCE foi de 0,75, IC 95\% (0,70-0,8I) para homens e 0,69, IC 95\% $(0,64-0,75)$ para mulheres. Os melhores pontos de coorte para discriminar o RCE foram para homens e mulheres, respectivamente: 0,52 (sensibilidade de 68\% e especificidade de 64\%) e 0,53 (sensibilidade de 67\% e especificidade de $58 \%)$.

ConcLusäo. Os resultados do estudo sugerem que a RCEst deve ser comparada aos demais indicadores antropométricos de obesidade e pode vir a ser utilizada para discriminar RCE.

UnItERMOS: Obesidade Central. Razão cintura-estatura. Risco coronariano.

\section{INTRODUÇÃo}

A obesidade é reconhecidamente um problema de saúde que afeta as sociedades em diversas partes do mundo, caracterizando-se na atualidade como uma epidemia com tendência a pandemia, não apenas em países industrializados, como também em locais menos desenvolvidos nas Américas Central e do Sul' e em outras regiões. Nos EUA, $61 \%$ da população apresentam, sobrepeso ou obesidade².

No Brasil, observa-se aumento nas prevalências da obesidade em inquéritos realizados em 1975, 1989 e 1996 para ambos os sexos e para todos os estratos socioeconômicos. Em estudo de revisão da literatura nacional, as prevalências de obesidade variaram entre $16 \%$ e $29,1 \%$.

Como fator de risco cardiovascular, o papel da obesidade é controverso, no entanto, a melhor explicação para a associação entre obesidade e doença cardíaca isquêmica é que esta ocorreria em um subgrupo de obesos, ou seja, nas pessoas que apresentassem adiposidade localizada na região abdominal ou central, mesmo na ausência da obesidade generalizada ${ }^{3}$.

Em pesquisas mais recentes, a gordura abdominal vem sendo considerada como forte fator de risco coronariano, em contraposição a diversos outros indicadores de obesidade como fator de risco cardiovascular já amplamente estudados ${ }^{4,5,6}$.

Existem diversos indicadores de obesidade total e central, porém o índice de massa corporal (IMC) é o indicador de obesidade total mais utilizado em estudos populacionais. Recente estudo demonstrou que o índice de conicidade (Índice C) é o indicador de obesidade central que melhor discrimina o elevado risco coronariano em pessoas do sexo masculino ${ }^{7}$. Outros autores ${ }^{8,9}$ demonstraram que a razão cintura-estatura (RCEst) é fortemente associada a diversos fatores de risco cardiovascular. Esses mesmos autores identificaram os pontos de coorte mais adequados deste indicador antropométrico de obesidade para discriminar o risco coronariano na China e em Taiwan ${ }^{8}$, sugerindo a utilização dos mesmos em estudos populacionais.

Assim, o objetivo deste estudo foi selecionar, por meio da sensibilidade e especificidade, os melhores pontos de coorte para a RCEst como discriminador de risco coronariano elevado (RCE) em adultos na cidade de Salvador-Brasil.

\section{Métodos}

Estudo transversal foi realizado em Salvador, Brasil, em 2000, em subgrupo de participantes do projeto "Monitoramento das Doenças Cardiovasculares e do Diabetes no Brasil" (MONIT), desenvolvido pela equipe de doenças crônicas não transmissíveis do Instituto de Saúde Coletiva (ISC) da Universidade Federal da Bahia (UFBA) e financiado pelo Ministério da Saúde do Brasil, que teve como objetivo, entre outros, determinar a prevalência dos fatores de risco cardiovascular na cidade de Salvador ${ }^{10}$. Para coleta dos dados do projeto MONIT, foi utilizado o censo domiciliar realizado por investigadores do projeto "Bahia Azul" em desenvolvimento por pesquisadores do ISC-UFBA, e direcionado a outros objetivos".

\section{Amostra}

A amostra foi probabilística por conglomerados em três etapas. Primeiro, os setores censitários de 8 das 10 bacias 
hidrográficas da cidade, com características sociodemográficas semelhantes, foram agrupados em "áreas de pesquisa" ( 108 áreas) e essas classificadas por nível socioeconômico (NSE) alto, misto e baixo. Ao todo, as áreas continham 16.592 domicílios, com aproximadamente 83.000 habitantes $\geq 20$ anos, sorteando-se probabilisticamente 37 delas, proporcionalmente ao número de setores de cada NSE. Nesse estágio, foram sorteados 1.540 domicílios, por amostra sistemática (intervalo $=10$ ), com resposta favorável à participação de 1.258 famílias $(81,7 \%)$ residentes em 63 setores censitários. No terceiro estágio, foram sorteados os participantes, no máximo dois por domicílio, um de cada sexo. Foram programadas 2.476 entrevistas, havendo $2,9 \%$ de recusas (72) e uma perda irrecuperável de 4,3\% (107) questionários completos. Dessa forma, a amostra analisada no projeto original foi constituída por 2.297 adultos com idade entre 20 a 74 anos, 1.025 do sexo masculino e 1.272 do sexo feminino. Como o modelo para cálculo do indicador de risco coronariano no presente estudo foi construído com base em população de 30 a 74 anos ${ }^{12}$, da amostra do projeto original foram excluídos os menores de 30 anos de idade, ficando então reduzida para 1.654 adultos, 7II homens e 943 mulheres. Todos os participantes deste subgrupo etário que completaram todo o protocolo de medidas proposto foram estudados, totalizando 391 homens e 577 mulheres, - que equivale a $55 \%$ de participantes do sexo masculino e $61 \%$ do sexo feminino em relação ao banco de dados original das faixas etárias em contexto.

\section{Coleta dos dados}

Todos os participantes da pesquisa foram entrevistados em domicílio para coleta dos dados demográficos, e tiveram a pressão arterial sistólica (PAS) e pressão arterial diastólica (PAD) medidas por seis vezes; as primeiras três, consecutivamente, 30 minutos após início da entrevista, e as três seguintes após intervalo de 20 minutos, braço esquerdo, sentado, bexiga vazia, sem ter fumado, tomado café ou álcool nos 30 minutos precedentes às tomadas. Das seis medidas de PAS e PAD, as primeiras foram excluídas e analisadas a média das cinco últimas. A cintura foi medida estando o participante na posição ereta, com o mínimo de roupa possível, na distância média entre a última costela flutuante e a crista ilíaca. A RCEst foi determinada por meio da divisão da circunferência da cintura $(\mathrm{cm})$ pela estatura $(\mathrm{cm})$. A medida da estatura foi feita no centro de saúde de cada bairro pela equipe do estudo, onde também foi coletado o sangue para os exames bioquímicos após 12 horas de jejum. Foram realizadas dosagens de colesterol total (método Trinder enzimático), HDLC (método Labtest) e glicemia (método Trinder enzimático). As técnicas e métodos para os exames bioquímicos seguiram a padronização da Sociedade Brasileira de Patologia Clínica. Dez entrevistadores de campo e duas supervisoras, ambas nutricionistas, foram devidamente treinados para todas as etapas do trabalho. Para testes e correções dos instrumentos e técnicas, inclusive da dinâmica do trabalho de campo, 50 residências ( 100 participantes) foram visitadas e as entrevistas e exames realizados seguiram toda a metodologia proposta. $\mathrm{O}$ grupo teste não se inclui na amostra. Para verificação das técnicas e instrumentos intra e interavaliadores foram feitas comparações entre médias, desvio-padrão e coeficientes de variação para medidas de circunferência, peso e estatura.

\section{Instrumentos utilizados}

Para medida da pressão arterial, foi utilizado tensiômetro eletrônico (OMRON, modelo HEM-705CP). A estatura foi medida com estadiômetro inglês, tipo Leicesters, acoplado a uma base que permite medida de altura em campo, fabricado pela Child Growth Foundation. O peso corporal foi medido em balanças tipo "banheiro", marca Filizola, com capacidade máxima de I50kg, aferidas pelo Instituto Nacional de Metrologia (INMETRO), com certificado próprio especificando margem de erro de \pm 100g. Para medida da circunferência de cintura foi utilizada fita métrica metálica e flexível marca Starrett, com definição de medida de $0,1 \mathrm{~cm}$.

\section{Variáveis de estudo}

Foram incluídas as seguintes variáveis: I) idade; 2) sexo; 3) estatura; 4) peso; 5) circunferência da cintura; 6) tabagismo; 7) glicemia; 8) colesterol total; 9) HDL-C; 10) PAS e II) PAD. Utilizando parte destas variáveis, foi construído um indicador de risco coronariano para representar em conjunto os fatores de risco cardiovascular analisados simultaneamente.

\section{Construção do indicador de risco coronariano}

Com base no acompanhamento por 12 anos de 2.489 homens e 2.856 mulheres de 30 a 74 anos, da coorte de Framingham, Wilson et al. ${ }^{12}$ construíram o algoritmo para medida do risco coronariano que serviu de modelo para o estudo de Salvador, Brasil. Durante o acompanhamento da coorte, 383 homens e 227 mulheres desenvolveram DAC. Através da utilização do modelo de regressão de Cox, os autores elaboraram uma tabela de pontuação (algoritmo) com a inclusão das variáveis por eles selecionadas (idade, PAS, PAD, colesterol total, HDL-C, tabagismo e diabetes). Para cada variável, a pontuação poderia ser positiva, quando considerada fator de risco, ou negativa, quando considerada fator de proteção. Para determinação dos pontos, foi utilizado o coeficiente beta dos modelos de análises de regressão de Cox'12.

Cada participante da amostra do presente estudo teve sua pontuação calculada com base no algoritmo supra citado. Para identificação do RCE, as somas das pontuações foram colocadas em ordem crescente e determinados os percentis, selecionando-se a soma da pontuação referente ao percentil 80 como de risco coronariano elevado, que correspondeu a 8 pontos para o sexo masculino e 10 pontos para o sexo feminino.

\section{Procedimentos de análise}

Os pontos de coorte para a RCEst foram determinados por meio das curvas ROC (Receiver Operating Characteristic). As curvas ROC foram originalmente desenvolvidas para uso com radar para separar a variabilidade de julgamento do observador da detectabilidade inata do sinal. Considerando a facilidade para 
distinguir entre o julgamento subjetivo e a detectabilidade objetiva, muitos investigadores têm sugerido sua aplicação em radiologia para avaliar a precisão do observador no sistema de imagens e comparar diagnósticos. Sugere-se também a aplicação das curvas ROC em estudos epidemiológicos para determinação de pontos de coorte em testes diagnósticos ou de triagem ${ }^{13}$.

Inicialmente, foi identificada a área total sob a curva ROC entre a RCEest e RCE. Utilizou-se intervalo de confiança (IC) a 95\%. Quanto maior a área sob a curva ROC, maior o poder discriminatório do indicador de obesidade para RCE. O IC determina se a capacidade preditiva do indicador de obesidade não é devido ao acaso. O limite inferior do IC não deve ser menor do que $0,50^{14}$.

$\mathrm{Na}$ seqüência, foram calculadas a sensibilidade e especificidade entre a RCEest e o RCE. Valores indicados por intermédio da curva ROC constituem pontos de coorte que deverão promover um mais adequado equilíbrio entre sensibilidade e especificidade para a RCEst como discriminador de RCE. Os dados foram analisados através do programa estatístico "STATA", versão 7.0. O projeto foi aprovado na íntegra pelo Comitê de Ética do Conselho Regional de Medicina do Estado da Bahia. Todos os participantes do estudo ou seus responsáveis assinaram termo de consentimento concordando em participar da pesquisa.

\section{Resultados}

As características da amostra estão demonstradas na Tabela I. Observa-se que, apesar de não existirem diferenças estatisticamente significativas entre homens e mulheres nas variáveis escolaridade, raça e risco coronariano, a maioria dos participantes da amostra apresenta baixo risco coronariano e não é branca. Observa-se também diferenças estatisticamente significativas entre homens e mulheres nas variáveis: peso corporal, estatura, circunferência da cintura, PAS, PAD, colesterol total, HDL-C e RCEest.

Na Figura I, pode-se observar a área sob a curva ROC entre RCEst e risco coronariano em pessoas do sexo masculino.

A área sob a curva ROC é de 0,76, IC 95\% (0,70-0,8I), sendo estatisticamente significativa. $O$ ponto de coorte de 0,52 apresenta 0 mais adequado equilíbrio entre sensibilidade (68\%) e especificidade (64\%) (Tabela 2). Neste ponto de coorte, o poder da RCEst para classificar corretamente pessoas do sexo masculino como RCE é de 68\% (sensibilidade), enquanto que o poder da RCEst para classificar corretamente pessoas do sexo masculino sem o risco coronariano é de 64\% (especificidade).

Por meio da Figura 2, pode-se observar a área sob a curva ROC entre a RCEst e risco coronariano em pessoas do sexo feminino.

A área sob a curva ROC é de 0,69, IC 95\% (0,64-0,75), sendo estatisticamente significativa. $O$ ponto de coorte de 0,53 apresenta o mais adequado equilíbrio entre sensibilidade (67\%) e especificidade (58\%) (Tabela 2). Neste ponto de coorte, o poder da RCEst para classificar corretamente pessoas do sexo feminino como RCE é de $67 \%$ (sensibilidade), enquanto que o poder da RCEst para classificar corretamente pessoas do sexo feminino sem o risco coronariano é de $58 \%$ (especificidade).
Tabela I - Média, desvio padrão, valores mínimos, máximos e percentuais das variáveis analisadas no estudo

\begin{tabular}{|c|c|c|c|}
\hline & Homens $(n=391)$ & Mulheres $(n=577)$ & pou $\chi^{2}$ \\
\hline $\begin{array}{l}\text { Idade } \\
\text { (anos) }\end{array}$ & $\begin{array}{l}45,3 \pm 10,4 \\
(30,0-74,0)\end{array}$ & $\begin{array}{l}45,7 \pm 11,6 \\
(30,0-74,0)\end{array}$ & 0,59 \\
\hline $\begin{array}{l}\text { Peso } \\
(\mathrm{Kg})\end{array}$ & $\begin{array}{c}68,9 \pm 12,3 \\
(39,1-124,5)\end{array}$ & $\begin{array}{l}64,2 \pm 13,8 \\
(37,0-143,6)\end{array}$ & 0,00 \\
\hline $\begin{array}{l}\text { Estatura } \\
(\mathrm{m})\end{array}$ & $\begin{array}{c}1,68 \pm 0,07 \\
(144,0-19 \mid, 0)\end{array}$ & $\begin{array}{c}1,55 \pm 0,07 \\
(100,0-181,0)\end{array}$ & 0,00 \\
\hline $\begin{array}{c}\text { Cintura } \\
(\mathrm{cm})\end{array}$ & $\begin{array}{c}85,6 \pm 10,1 \\
(62,0-122,8)\end{array}$ & $\begin{array}{c}82,7 \pm 12,2 \\
(43,8-129,3)\end{array}$ & 0,00 \\
\hline $\begin{array}{c}\text { PAS } \\
(\mathrm{mmHg})\end{array}$ & $\begin{array}{l}130,6 \pm 22,8 \\
(81,5-238,2)\end{array}$ & $\begin{array}{l}124,8 \pm 24,6 \\
(82,2-229,6)\end{array}$ & 0,00 \\
\hline $\begin{array}{l}\text { PAD } \\
(\mathrm{mmHg})\end{array}$ & $\begin{array}{l}81,1 \pm 14,6 \\
(55-143,6)\end{array}$ & $\begin{array}{c}78,2 \pm 13,2 \\
(50-139)\end{array}$ & 0,00 \\
\hline $\begin{array}{l}\text { Colesterol total } \\
(\mathrm{mg} / \mathrm{dl})\end{array}$ & $\begin{array}{c}220,6 \pm 52,0 \\
(102-522) \\
\end{array}$ & $\begin{array}{c}232,3 \pm 52,5 \\
(123-468)\end{array}$ & 0,00 \\
\hline $\begin{array}{l}\text { HDL-C } \\
(\mathrm{mg} / \mathrm{dl})\end{array}$ & $\begin{array}{c}48,5 \pm 13,1 \\
(24-114) \\
\end{array}$ & $\begin{array}{c}51,5 \pm 13,5 \\
(26-98) \\
\end{array}$ & 0,00 \\
\hline $\begin{array}{c}\text { Glicemia } \\
(\mathrm{mg} / \mathrm{dl})\end{array}$ & $\begin{array}{c}90,5 \pm 29,2 \\
(61-308) \\
\end{array}$ & $\begin{array}{c}92,0 \pm 34,4 \\
(59-318) \\
\end{array}$ & 0,48 \\
\hline RCEst & $\begin{array}{l}0,51 \pm 0,06 \\
(0,36-0,69)\end{array}$ & $\begin{array}{l}0,53 \pm 0,08 \\
(0,27-0,84)\end{array}$ & 0,00 \\
\hline $\begin{array}{l}\text { Risco coronarian } \\
\text { Não elevado } \\
\text { Elevado }\end{array}$ & $\begin{array}{l}76 \% \\
24 \% \\
\end{array}$ & $\begin{array}{l}79 \% \\
21 \% \\
\end{array}$ & 0,61 \\
\hline $\begin{array}{c}\text { Escolaridade } \\
\text { Baixa } \\
\text { Média/Alta }\end{array}$ & $\begin{array}{l}50 \% \\
50 \%\end{array}$ & $\begin{array}{l}54 \% \\
46 \%\end{array}$ & 0,57 \\
\hline $\begin{array}{c}\text { Raça } \\
\text { Brancos } \\
\text { Negros e outros }\end{array}$ & $\begin{array}{l}24 \% \\
76 \%\end{array}$ & $\begin{array}{l}26 \% \\
74 \%\end{array}$ & 0,74 \\
\hline
\end{tabular}

Valores contínuos foram comparados através do teste "t" de Studant para amostras independentes e valores percentuais por meio do teste Qui-quadrado; PAS, pressão arterial sistólica; PAD, pressão arterial distólica; HDL-C, lipoproteína de alta densidade; RCEst, razão cintura-estatura; escolaridade baixa, analfabeto/nunca foi à escola, porém lê e escreve; escolaridade média/alta, $1^{\circ}, 2^{\circ}$ grau e universitário; $\chi^{2}$, teste do Qui-quadrado.

\section{Discussão}

Diversos estudos têm demonstrado que a RCEst é um bom discriminador de obesidade abdominal relacionada a fatores de risco cardiovascular, bem como de RCE $8,9,15$.

Em estudo realizado com amostra de 55.563 adultos de ambos os sexos em Taiwan, com objetivo de identificar os pontos de coorte da RCEst para discriminar pelo menos um fator de risco cardiovascular (diabetes, hipertensão ou dislipidemia), encontrou-se valores de 0,48 e 0,45 para homens e mulheres, respectivamente ${ }^{8}$.

Ainda em Taiwan, utilizando-se como amostra 38.556 sujeitos de ambos os sexos observou-se forte associação da RCEst com hipertensão arterial, intolerância à glicose, diabetes e dislipidemias ${ }^{15}$. 


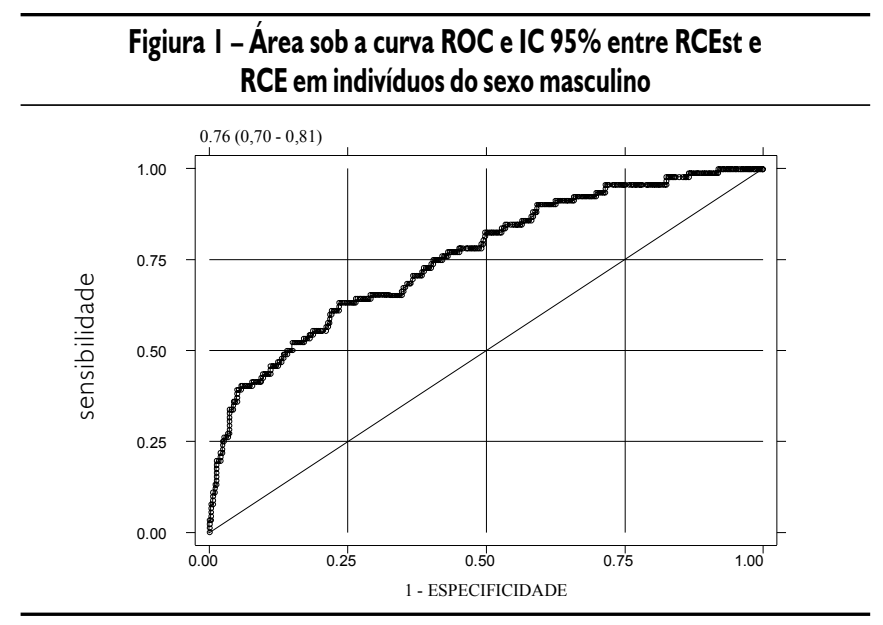

Tabela 2 - Pontos de coorte, sensibilidade e especificidade da RCEst como discriminador de RCE

\begin{tabular}{lccc}
\hline RCEst & Ponto de coorte & Sensibilidade & Especificidade \\
Masculino & 0,52 & $68 \%$ & $64 \%$ \\
Feminino & 0,53 & $67 \%$ & $58 \%$ \\
\hline
\end{tabular}

RCEst, razão cintura-estatura; RCE, risco coronariano elevado

Figura 2 - Área sob a curva ROC e IC $95 \%$ entre RCEst e RCE em indivíduos do sexo feminino

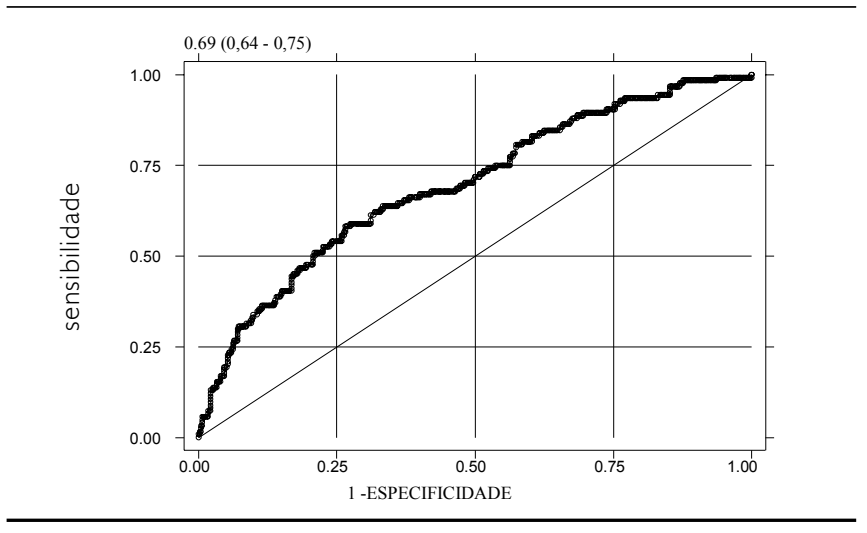

Em estudo realizado na cidade do México, Berber et al. ${ }^{16}$ observaram pontos de coorte variando entre 0,525 e 0,535 para RCEst como discriminador de diabetes, hipertensão e dislipidemias. Também na China, em pesquisa realizada com 2.895 pessoas com idade variando entre 25-74 anos, observou-se que a RCEst é o melhor indicador antropométrico de obesidade para discriminar fatores de risco cardiovascular. O ponto de coorte mais adequado para este objetivo é 0,48 , tanto para homens quanto para mulheres?

Nos estudos citados anteriormente, os pontos de coorte identificados referem-se a fatores de risco coronarianos analisados isoladamente. No nosso trabalho, a análise da variável resposta foi realizada por meio da construção de indicador de risco coronariano usando o algoritmo proposto com base na coorte de Framingham ${ }^{2}$. Os valores encontrados ficaram próximos aos identificados nos estudos citados (0,52 e 0,53 para homens e mulheres, respectivamente).

A classificação do RCE usando o algoritmo pode ter sido uma limitação do estudo. Utilizamos este algoritmo aplicando seus valores nos indivíduos participantes da nossa amostra e definimos que o RCE seria identificado por pontuação referente ao percentil 80 na distribuição de percentis da própria populaçãa de estudo. Por outro lado, existem controvérsias quanto à pontuação ideal para identificação do RCE em curto prazo. Alguns autores ${ }^{17}$ sugerem que o RCE poderia ser identificado através da pontuação equivalente a 20\% do risco absoluto para coronariopatia nos próximos 10 anos, o que equivaleria a 9 pontos para homens e 15 pontos para mulheres no algoritmo proposto com base na coorte de Framingham ${ }^{2}$. No nosso estudo, o RCE foi identificado por meio do ponto de coorte de 8 pontos para homens e 10 pontos para mulheres.

Estudos recentes demonstram que a predição do RCE em longo prazo feita por meio do algoritmo proposto com base na coorte de Framingham ${ }^{12}$ pode ser generalizada para outras populações, bem como pode ser utilizada em estudos transversais. A generalização para outras populações deve ser feita após recalibração para diferentes prevalências dos fatores de risco envolvidos na análise ${ }^{18,19}$.

Por outro lado, os resultados encontrados neste trabalho são oriundos de adultos de ambos os sexos com idade maior ou igual a 30 anos, recomendando-se cautela ao serem usados em adultos com menos de 30 anos de idade. Deve-se considerar, também, que existem modificações na composição corporal com o processo do envelhecimento, o que poderia gerar pontos de coorte da RCEst para diferentes grupos etários.

Para o presente estudo, o subgrupo estudado foi constituído por pessoas que compareceram para completar o protocolo de medidas proposto. Isto poderia ser atribuído a diferenças entre características dos sujeitos que efetivamente participaram do estudo e os não participantes. Para esclarecer esta possibilidade, foi realizada comparação entre o dois grupos, observando-se que apenas na variável PAS, para ambos os sexos, houve diferença estatisticamente significativa na média entre os grupos, porém ao ser feita análise das proporções observouse que a prevalência de níveis elevados de PAS não apresenta diferença. Apenas na variável escolaridade para o sexo feminino houve diferença estatisticamente significativa entre os grupos dos incluídos e não inclúidos. Assim, entendemos que, apesar da redução no tamanho da amostra, provavelmente não aconteceu viés de seleção.

\section{Conclusão}

Os resultados sugerem que a RCEst pode vir a ser utilizada para discriminar alto risco coronariano, apesar dos valores de sensibilidade e especificidade não serem muito elevados. Considerando que os melhores pontos de coorte encontrados foram de 0,52 para homens e 0,53 para mulheres, a mensagem de saúde pública poderia ser de que a circunferência da cintura de determinada pessoa não deve ser maior do que a metade da sua estatura. Além disto, sugerem-se novos estudos que possam comparar a RCEst com outros indicadores de obesidade mais comumente utilizados para discriminar RCE. Sugere-se também estudos com crianças, adolescentes e adultos de diferentes grupos etários para que se possa observar o poder discriminatório da RCEst para RCE nestes subgrupos específicos. 
Conflito de interesse: Trabalho financiado pela Secretaria de Vigilância em Saúde, Ministério da Saúde e Banco Mundial

\section{SUMMARY}

\section{WAIST-TO-HEIGHT RATIO AS A CORONARY RISK PREDICTOR AMONG ADULTS}

BACKGROUND. This study proposes to identify the sensibility, specificity and the best cut-off point for waist-to-height ratio (WHtR) as a predictor of coronary high risk (CHR).

METHODS. A cross-sectional study was applied to a population comprised of 968 adults between 30 and 74 years of age, of which 391 (40.4\%) were male. Receiver Operating Characteristic (ROC) curves were employed to identify the sensibility and specificity of the best cut-off point for WHtR as a predictor of CHR. The statistical significance of the area under ROC curve was also verified using a confidence interval (Cl) of $95 \%$.

RESULTS. The totalarea underROC curve between WHtRand coronary riskwas $0.75, \mathrm{Cl} 95 \%(0.70-0.81)$ formen and $0.69, \mathrm{Cl} 95 \%(0.64-0.75)$ foromen. For men, 0.52 was found to be the best cut-off point for predicting CHR (sensibility 68\% and specificity 64\%). Among women, 0.53 was the best cut-off point for discriminating CHR (sensibility $67 \%$ and specificity $58 \%$ ).

ConCLUSIONS. Results suggest that the WHtR may be employed to identify CHRand must be compared to other anthropometric indicators of obesity. [Rev Assoc Med Bras 2006; 52(3): |57-6I]

KEY WORDS: Central obesity. Waist-to-Height ratio. Coronary risk.

\section{REFERÊNCIAS}

I. Heseker H, Schmid A. Epidemiology of obesity. Ther Umsch 2000;57(8):478-81.

2. Wyatt HR. The prevalence of obesity. Prim Care 2003;30(2):267-79.

3. Lessa I. O adulto brasileiro e as doenças da modernidade: epidemiologia das doenças crônicas não-transmissíveis. São Paulo: Editora HucitecAbrasco; 1998.

4. Gustat J, Elkasabany A, Srinivasan S, Bereson S. Relation of abdominal heigth to cardiovascular risk factors in young adults. Am J Epidemiol 2000; I5I(9):885-91.

5. Visscher TI, Seidell JC, Molarius A, Van Der Kuip D, Hofman A, Witteman JC. A comparison of body mass index, waist-hip ratio and waist circumference as predictors of all-cause mortality among the elderly: The Rotterdam study. Int J Obes Relat Metab Disord 200 I; 25(I): I 730-5.

6. Poulter N. Global risk of cardiovascular disease. Heart 2003;89(Suppl 2): $112-5$
7. Pitanga FJG, Lessa I. Indicadores antropométricos de obesidade como instrumento de triagem para risco coronariano elevado em adultos na cidade de Salvador-Bahia. Arq Bras Cardiol 2005; 85(I): 26-3I.

8. Lin WY, Lee LT, Chen CY, Lo H, Hsia HH, Liu IL, et al. Optimal cut-off values for obesity: using simple anthropometric indices to predict cardiovascular risk factors in Taiwan. Int J Obes Relat Metab Disord 2002;26(9): 1232-8.

9. Ho SY, Lam TH, Janus ED. Waist to stature ratio is more strongly associated with cardiovascular risk factors than other simple anthropometric indices. Ann Epidemiol 2003; | 3( | 0):683-9 |

10. Brasil. Ministério da Saúde. Centro Nacional de Epidemiologia (CENEPI). Instituto de Saúde Coletiva (ISC). Projeto monitoramento de doenças cardiovasculares e do diabetes no Brasil (MONIT). Relatório. Brasília: Ministério da Saúde - CENEPI; 2000.

II. Teixeira MG, Barreto, ML, Costa MCN, Strina, A, Martins Júnior D, Prado, M. Áreas sentinelas: uma estratégia de monitoramento em saúde pública. Cad Saúde Pública 2002; | 8(5): I | 89-95.

12. Wilson PWF, D'agostino RB, Levy D, Belanger AM, Silbershatz H, Kannel WB. Prediction of coronary heart disease using risk factores categories. Circulation 1998;97: 1837-47.

13. Erdreich LS, Lee ET. Use of relative operating characteristics analysis in epidemiology: a method for dealing with subjective judgement. Am J Epidemiol 1981; | | 4(5):649-62.

14. Schisterman EF, Faraggi D, Reiser B, Trevisan M. Statistical inference for the area under the receiver operating characteristic curve in the presence of random measurement error. Am J Epidemiol 200 I; I 54(2): I 74-9.

15. Huang KC, Lin WY, Lee LT, Chen CY, Lo H, Hsia HH et al. Four anthropometric indices and cardiovascular risk factors in Taiwan. Int J Obes Relat Metab Disord 2002;26(8): 1060-8.

16. Berber A, Gomez-Santos R, Fanghanel G, Sanchez-Reyes L. Anthropometric indexes in the prediction of type 2 diabetes mellitus, hypertension and dyslipidaemia in a mexican population. Int J Obes Relat Metab Disord 200 I;25( I 2): I794-9.

17. Grundy SM, Pasternak R, Greenland P, Smith S, Fuster V. Assessment of cardiovascular risk by use of multiple-risk-factor assessment equations. J Am Coll Cardiol 1999;34(I0): 1348-59.

18. D'Agostino RB, Grundy S, Sullivan LM, Wilson P. Validation of the Framingham coronary Herat disease prediction scores. JAMA 200 I;286(2): I 80-7.

19. Cappuccio FP, Oakeshott P, Kerry SM. Application of Framingham risk estimates to ethnic minorities in United Kingdom and implications for primary prevention of heart disease in general pratic: cross sectional population based study. BMJ 2002;325( | 2): | 27| -80.

Artigo recebido: 10/02/2005

Aceito para publicação: 28/06/2005 